\section{Uncoupling pain from the psyche}

\section{By Tim Fulmer, Senior Writer}

University of Toronto researchers have reported a way to block paininduced activation of neuronal receptors while preserving their basic functions. ${ }^{1}$ The strategy could avoid the psychomotor side effects associated with centrally acting chronic pain drugs such as $\mathrm{N}$-methyl$\mathrm{D}$-aspartic acid receptor antagonists. The researchers have already founded a biotech, NoNO Inc., to advance the strategy.

Chronic neuropathic pain is caused by damage to the peripheral or central nervous systems. Unlike acute pain, which is self-limiting and self-resolving, neuropathic pain can persist for decades after the initial injury. Its hallmarks are allodynia, which is pain in response to a usually nonpainful stimulus like a light touch, and hyperalgesia, which is abnormally increased sensitivity to a painful stimulus.

The established role of $N$-methyl-D-aspartic acid receptors (NMDA receptors) in central mechanisms of neuropathic pain suggests that blocking them would be an ideal way to manage pain. However, the receptors are expressed in many regions of the CNS, where they play important roles in a variety of key neurological processes. Thus, although directly antagonizing the receptors has shown benefit in treating neuropathic pain, the associated behavioral and sensory disturbances can lead to unacceptable side effects.

In their Nature Medicine paper, Michael Salter and colleagues at the University of Toronto sought to inhibit mechanisms that upregulate the NMDA receptor in pain without blocking the receptor's channel, through which cation currents flow that potentially play a role in learning and memory.

To do so, the researchers designed an uncoupling peptide that bound the surface of the NMDA receptor inside the cell and prevented it from interacting with v-src sarcoma (Schmidt-Ruppin A-2) viral oncogene homolog (avian) (SRC), an intracellular protein tyrosine kinase that enhances NMDA receptor activity in pain, epilepsy and some neurodegenerative disorders (see Figure 1, "Uncoupling neuropathic pain"). ${ }^{2}$

To get the peptide into the cell, the team fused it to the protein transduction domain (PTD) of the HIV-1 Tat protein, which has been used by many labs to deliver a variety of proteins across cell membranes in vivo. ${ }^{3}$ Though derived from a viral protein, the PTD is not an infectious agent.

In two rat models of chemically induced inflammatory pain, i.v. delivery of the uncoupling peptide lowered pain hypersensitivity compared with the effect of saline controls.
In a rat model of neuropathic pain, the peptide significantly lowered tactile and cold hypersensitivity compared with the effect of saline control $(p<0.05)$. Src knockout in those rats led to significant decreases in hypersensitivity compared with that seen in wild-type littermates $(p<0.01)$ and similar decreases compared with those seen using the uncoupling peptide, indicating that the peptide's mechanism was indeed Src-dependent.

Finally, the uncoupling peptide caused no significant behavior alterations in two different mouse models of NMDA receptormediated learning and memory: contextual fear conditioning and conditioned taste aversion.

"Our findings reveal a strategy relevant to the treatment of chronic pain and potentially other NMDA receptor-dependent central nervous system pathologies," the authors concluded.

"The NMDA receptor plays a well-established role in mediating neuropathic pain. However, highly efficacious NMDA receptor antagonists like ketamine have serious psychoactive side effects that make them generally undesirable for treating pain outside of severe, acute trauma," Clifford Woolf of Harvard Medical School told SciBX. "The Nature Medicine paper now illustrates an alternative strategy that potentially preserves the efficacy of NMDA receptor antagonists while significantly reducing those side effects."

Woolf is professor of anesthesiology and director of the Neural Plasticity Research Group at Massachusetts General Hospital and Harvard Medical School, and is himself cofounder of a company developing a pain therapeutic.

\section{Optimizing the strategy}

Salter and a colleague of his at Univeristy of Toronto, Michael Tymianski, founded NoNO to identify peptide and small molecule inhibitors of NMDA receptor-SRC interactions and additional protein-protein interactions to treat pain, stroke and other neurological disorders.

Salter is senior scientist at the Hospital for Sick Children, director of the Centre for the Study of Pain and professor of physiology at the University of Toronto. Tymianski is president and CEO of NoNO, professor of surgery and physiology at the university and senior scientist at the Toronto Western Hospital Research Institute.

NoNO's lead compound is NA-1, a peptide that inhibits interactions between the NMDA receptor and discs, large homolog 4 (Drosophila) (DLG4; PSD95), a scaffold protein that is a binding partner of SRC at the postsynaptic terminal. ${ }^{4,5}$ The compound is in Phase IIb testing to treat stroke in patients undergoing aneurysm repair. According to Tymianski, NA-1 has also shown efficacy in neuropathic pain models.

Salter told SciBX he is planning preclinical studies of an NMDA receptor-SRC uncoupling peptide to optimize dosing and establish a toxicology profile. In the longer term, he hopes to study the peptide or a small molecule analog in patients.

"Since neuropathic pain is often a chronic condition requiring long-term treatment, it's probably worth looking for orally active small 
molecule compounds that do the same thing as the peptide," noted Tymianski.

A patent on the NMDA receptor-SRC uncoupling peptide strategy is held by the Hospital for Sick Children and is licensed to NoNO.

\section{Multiple mechanisms}

Given the complexity of the peripheral and central mechanisms involved in neuropathic pain, companies polled by SciBX had mixed opinions on how essential targeting the NMDA receptor pathway is to treating neuropathic pain.

Chris O'Brien, CMO and SVP of clinical development at Neurocrine Biosciences Inc., wanted to see additional evidence that the uncoupling peptide strategy had indeed lowered off-target effects on learning and memory compared with using conventional NMDA receptor antagonists like ketamine.

The problem, according to O'Brien, is that neuropathic pain has both a peripheral and a central component. Targeting the former may not be sufficient to block CNS pain sensitization mechanisms. However, targeting the latter can carry the risk of seriously impairing cognitive and behavioral functions, he said.

Neurocrine has an undisclosed selective norepinephrine reuptake inhibitor that has completed a Phase I safety trial for neuropathic pain and multiple ion channel blockers in preclinical testing to treat chronic pain.

Even within the CNS, the role glial cells play in the development of chronic pain ${ }^{6,7}$ suggests that solely targeting neuronal pathways may not always be sufficient to attenuate pain.

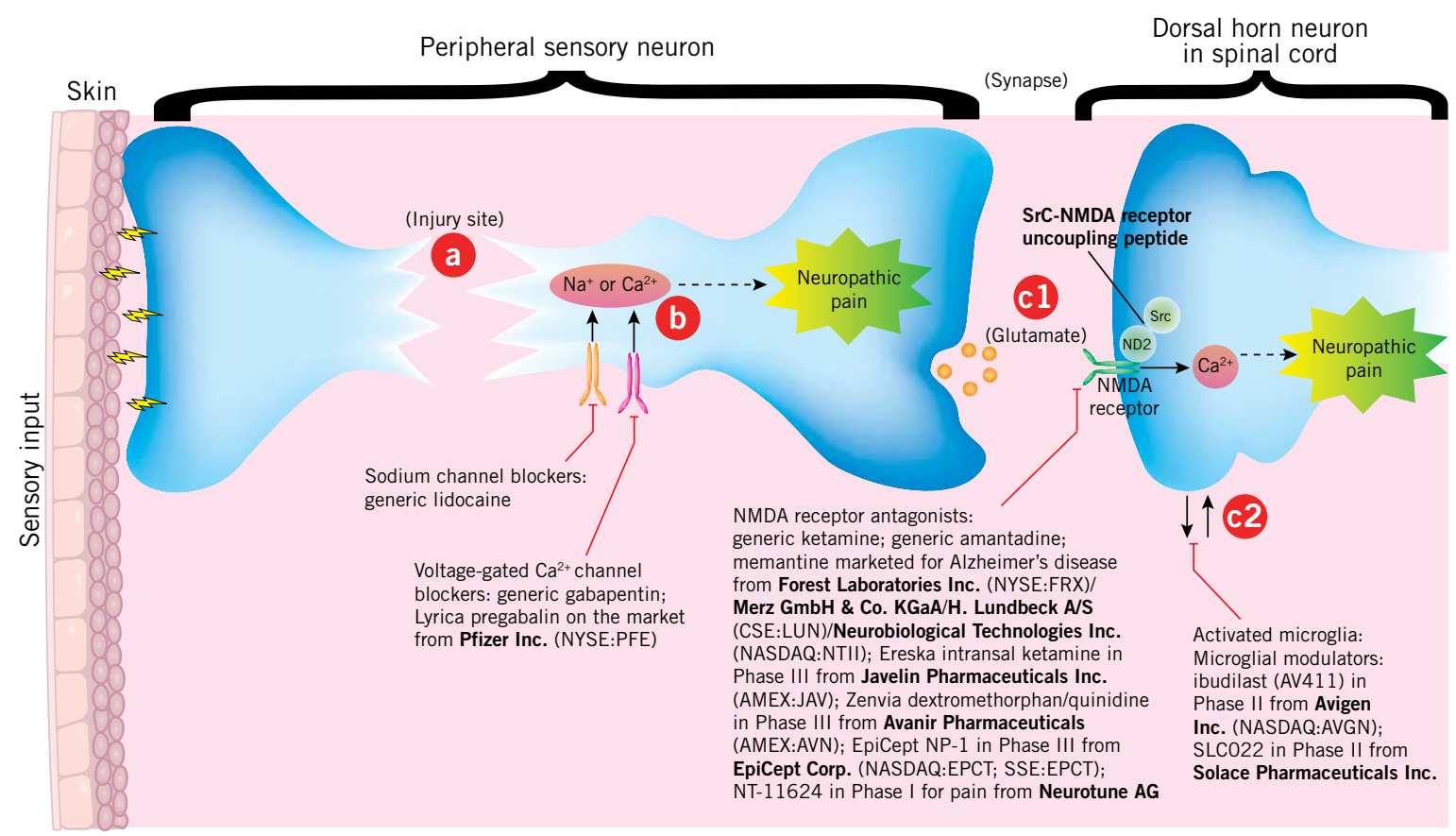

Figure 1. Uncoupling neuropathic pain. Most forms of chronic pain are neuropathic in nature and characterized by damage to the peripheral and/or central nervous systems. Strategies for treating and managing neuropathic pain thus typically target mechanisms in peripheral sensory neurons or spinal cord dorsal horn neurons-a new therapeutic approach to the latter is highlighted in Nature Medicine. ${ }^{1}$

[a] Injury and trauma to a peripheral nerve leads to the release of multiple cytokines, chemokines and other inflammatory mediators that bind receptors on both damaged and intact neurons, as well as the invasion of immune cells, triggering multiple pathological mechanisms.

[b] One of those mechanisms involves the overactivation of ion channels in sensory neurons, with consequent increased neuronal firing and neurotransmitter release at the synaptic terminals - all of which contribute to the development of acute neuropathic pain. At this stage, sodium channel blockers and voltage-gated calcium channel blockers can help minimize pain.

Neurotransmitters and inflammatory mediators released by the injured peripheral sensory neurons can also act on cells in the CNS, including dorsal horn neurons and microglia.

[c1] Glutamate released by the presynaptic terminal activates $N$-methyl-D-aspartic acid receptors (NMDA receptors), leading to higher calcium levels, increased neuronal firing and central neuropathic pain. In the Nature Medicine paper, researchers show that blocking paininduced NMDA receptor activation at this step rather than directly antagonizing the receptor can potentially decrease central neuropathic pain with fewer psychoactive side effects compared with using conventional NMDA receptor antagonists. Researchers blocked NMDA receptor activation using a peptide that prevents interactions between v-src sarcoma (Schmidt-Ruppin A-2) viral oncogene homolog (avian) (SRC) and the ND2 subunit of the NMDA receptor.

[c2] By another mechanism, microglia in the vicinity of the dorsal horn neurons can become activated, releasing proinflammatory factors that act on the neuron and potentially increase the pain.

Unless noted otherwise in the figure, the compounds listed are indicated for pain. 
"The mechanistic complexity underlying neuropathic pain means that, much like current standard of care, next-generation pain therapeutics will likely be used in a combinatorial setting," said Woolf, who is a cofounder of Solace Pharmaceuticals Inc. "Thus, a compound that modulates synaptic plasticity, like Salter's uncoupling peptide, could conceivably be combined with a small molecule compound that modulates the activity of proinflammatory glial cells in the CNS."

Kirk Johnson, VP of R\&D at Avigen Inc., agreed. "A multi-pronged approach may be advantageous for therapy of chronic pain syndromes," he told SciBX.

Solace is developing SLC022, a small molecule glial cell modulator that is in Phase II testing to treat neuropathic pain. Avigen's Ibudilast (AV411), a small molecule glial attenuator that suppresses release of the proinflammatory cytokines IL- $1 \beta$, tumor necrosis factor- $\alpha$ and IL- 6 by glial cells, is in Phase II testing for neuropathic pain.

The existence of substantive neuron-glia crosstalk suggests that a robust therapeutic strategy in neuropathic pain would probably need to target paininduced mechanisms in both neurons and glial cells, noted Ru-Rong Ji, associate professor of anesthesiology and associate director of the Pain Research Center at Brigham and Women's Hospital and Harvard Medical School.

"We've found that three different MAP [mitogen-activated protein] kinases-p38, JNK and ERK-are activated in spinal glial cells following injury to the axons of peripheral sensory neurons. Growing evidence suggests that these alterations in glial signaling subsequently act back on the spinal neurons and potentially contribute to the development and maintenance of chronic neuropathic pain," Ji told SciBX.

\section{Sticking with neurons}

Other researchers expect that focusing primarily on neuronal mechanisms in pain could be fruitful if cognitive side effects can be avoided.

One potential strategy would be to specifically target neuronal pain generators in the periphery, said Keith Bley, SVP of nonclinical R\&D at NeurogesX Inc.

The company is seeking to do that with NGX-4010, a dermal patch containing capsaicin, a transient receptor potential vanilloid 1 (TRPV1; VR1) agonist. The goal is to produce a persistent decrease in the hyperactivity of sensory nerve fibers that serve as peripheral pain generators. The capsaicin delivered by the patch works locally on the skin's nociceptive sensory nerve fibers, typically below dose levels that could lead to CNS and gastrointestinal adverse effects, said Bley.

NeurogesX has submitted an NDA for NGX-4010 to treat pain associated with post-herpetic neuralgia. The compound is also in Phase III testing to treat pain associated with HIV distal sensory polyneuropathy.

EpiCept Corp. is pursuing a similar strategy with its EpiCept NP-1, a topical cream containing $4 \%$ amitriptyline, an antidepressant, and $2 \%$ ketamine, an NMDA receptor antagonist.

The topical approach should result in generally lower plasma levels of the NMDA receptor antagonist than systemically administered drug and thus should have a lower side effect profile, said Jana Sawynok, co-inventor of NP-1 and chairman of pharmacology at Dalhousie University.

Moreover, according to Sawynok, because the cream has two active ingredients, it may have the additional advantage of targeting interactions at multiple steps in the pain signaling pathway.

NP-1 is in Phase III testing for chemotherapy-induced peripheral neuropathy, Phase IIb testing for diabetic peripheral neuropathy and Phase
IIb testing for peripheral herpetic neuropathy.

At least two other biotechs are hoping to limit antagonism to specific subtypes of receptors expressed on CNS neurons and thus potentially avoid the sedative and motor side effects that often occur with the global antagonism of some neuronal receptors.

Neurotune AG thinks that directly targeting NMDA receptors is still the best way to treat some forms of neuropathic pain. The company expects that highly selective antagonism of NMDA receptors can avoid unwanted side effects while maintaining efficacy.

Neurotune's NT-11624, an orally active subtype-selective NMDA receptor antagonist, has completed a Phase I safety trial in healthy volunteers. "In that trial, at the rather high doses we studied, we saw no signs of psychoactive side effects like psychosis and memory and motor deficits," said CEO Andreas Schulze.

Schulze said the company now plans to move NT-11624 into Phase II testing to treat HIV-associated pain and also osteoarthritis pain. "We've chosen our subindications of neuropathic pain based on NT-11624's efficacy profile in a range of preclinical neuropathic pain models," he said.

The company has not disclosed what region of the NMDA receptor complex NT-11624 binds.

Similarly, NeuroSearch A/S is targeting subtypes of the $\gamma$-aminobutyric acid $A\left(\mathrm{GABA}_{\mathrm{A}}\right)$ receptor, which binds the principal inhibitory neurotransmitter of the spinal cord.

"The strategy here is to design modulators of the $\mathrm{GABA}_{\mathrm{A}}$ receptor that have selective affinity for receptors composed of particular subunits. By thus modulating a restricted subset of the receptors in the CNS, we can potentially treat neuropathic pain without unwanted sedation, motor impairment and tolerance development," said Gordon Munro, scientific officer at NeuroSearch.

One of the modulators, NS11394, has shown efficacy in rat models of inflammatory and neuropathic pain without sedation or motor impairment. ${ }^{8}$

Fulmer, T. SciBX 1(45); doi:10.1038/scibx.2008.1090

Published online Dec. 18, 2008

\section{REFERENCES}

1. Liu, X. et al. Nat. Med.; published online Nov. 16, 2008; doi:10.1038/nm.1883

Contact: Michael Salter, University of Toronto, Toronto, Ontario, Canada e-mail: Mike.salter@utoronto.ca

2. Salter, M. \& Kalia, L. Nat. Rev. Neurosci. 5, 317-328 (2004)

3. Chauhan, A. et al. J. Control. Release 117, 148-162 (2007)

4. Cui, H. et al. J. Neurosci. 27, 9901-9915 (2007)

5. Kalia, L. et al. EMBO J. 25, 4971-4982 (2006)

6. Scholz, J. \& Woolf, C. Nat. Neurosci. 10, 1361-1368 (2007)

7. Watkins, L. \& Maier, S. Nat. Rev. Drug Discov. 2, 973-985 (2003)

8. Munro, G. et al. J. Pharmacol. Exp. Ther. 327, 969-981 (2008)

\section{COMPANIES AND INSTITUTIONS MENTIONED}

Avigen Inc. (NASDAQ:AVGN), Alameda, Calif.

Brigham and Women's Hospital, Boston, Mass.

Dalhousie University, Halifax, Nova Scotia, Canada

EpiCept Corp. (NASDAQ:EPCT; SSE:EPCT), Tarrytown, N.Y.

Harvard Medical School, Boston, Mass.

Hospital for Sick Children, Toronto, Ontario, Canada

Massachusetts General Hospital, Boston, Mass.

Neurocrine Biosciences Inc. (NASDAQ:NBIX), San Diego, Calif.

NeurogesX Inc. (NASDAQ:NGSX), San Carlos, Calif.

NeuroSearch A/S (CSE:NEUR), Ballerup, Denmark

Neurotune AG, Schlieren-Zurich, Switzerland

NoNO Inc., Toronto, Ontario, Canada

Solace Pharmaceuticals Inc., Boston, Mass.

Toronto Western Hospital Research Institute, Toronto, Ontario, Canada University of Toronto, Toronto, Ontario, Canada 